\title{
Noninvasive Detection of Differential Water Content Inside Biological Samples Using Deep Raman Spectroscopy
}

\author{
Adrian Ghita, Thomas Hubbard, Pavel Matousek,* and Nicholas Stone* \\ Cite This: Anal. Chem. 2020, 92, 9449-9453 \\ Read Online
}

ABSTRACT: Here we conceptually demonstrate the capability of deep Raman spectroscopy to noninvasively monitor changes in the water content within biological tissues. Water was added by injection into an isolated tissue volume (a $20 \mathrm{~mm}$ diameter disk of $5 \mathrm{~mm}$ thickness) representing a $20 \%$ increase in the overall mass, which was equivalent to a $5 \%$ increase in the water/tissue content. The elevated water content was detected through a larger volume of tissue with a total thickness of approximately $12 \mathrm{~mm}$ and a spiked tissue segment located in its center using transmission Raman spectroscopy (TRS) by monitoring the change of the $\mathrm{OH}\left(\sim 3390 \mathrm{~cm}^{-1}\right)$ Raman band area (3350-3550 $\mathrm{cm}^{-1}$ spectral region) after being normalized to the neighboring $\mathrm{CH}$ stretching band. The tissue sample was raster scanned with TRS to yield a spatial map of the water concentration within the sample encompassing the spiked tissue zone. The mapping revealed the presence and location of the

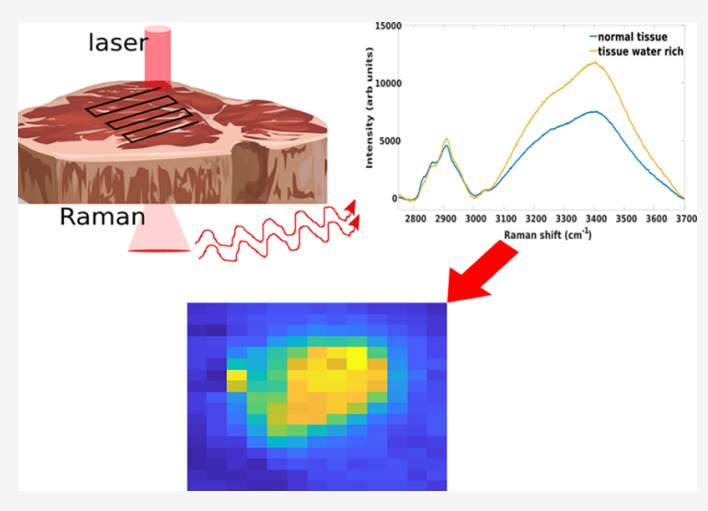
spiked region. The results provide the first conceptual demonstration using a

deep Raman-based architecture, which can be used noninvasively for the detection of an elevated water content deep within biological tissues. It is envisaged that this concept could play a role in rapid in vivo detection and localization of cancerous lesions (generally exhibiting a higher water content) beneath the tissue surface.

$\mathrm{B}$ iological tissues naturally contain a high amount of water, playing a vital role in the overall biochemical landscape of all living organisms. Local water concentration has been found to vary with the local biochemical environment, such as the presence of invasive malignancies. ${ }^{1}$ Water accumulation in cancerous tissue may be a consequence of the presence of diffusion barriers, ${ }^{2}$ an accumulation of transmembrane proteins ${ }^{3}$ in cell membranes or extracellular compartments, preventing the balancing of fluids that leak out of the poorly formed neovasculature associated with invasive tumor sites.

The noninvasive measurement of the water content in the human body can be accomplished by MRI; ${ }^{4}$ however, this is reliant upon expensive and complex infrastructure. The utilization of near-infrared absorption spectroscopy represents another alternative as it allows penetration depths on the order of several centimeters to be achieved through tissues; ${ }^{5}$ however, it yields relatively low chemical specificity due to typically broad and overlapping spectral features. ${ }^{6}$ The use of Raman spectroscopy, a technique with an inherently high molecular specificity, offers an alternative solution. Nearinfrared confocal Raman spectroscopy has been demonstrated to monitor skin moisture levels at depth levels of 200-300 $\mu \mathrm{m}$. Furthermore, the method does not require sample preparation and is easily implementable in vivo at tissue surfaces either through skin or using probes to reach the lining of hollow organs.
In the surgical excision of solid organ tumors, the distance between the edge of the tumor and the edge of the resected specimen or "surgical margin" is of prognostic significance. If the surgical margin is too small, it is an inadequate resection, leading to re-excision procedures associated with a lower survival rate and cosmetic outcome, anxiety, and additional medical costs. ${ }^{9,10}$ In earlier pioneering studies, a Raman water peak, detected in the high-wavenumber spectral region, was used as a biomarker for the localization of head and neck squamous cell carcinoma (SSC). Monitoring of the relative water to protein concentration ( $\mathrm{OH}$ to $\mathrm{CH}$ peaks) has led to the development of a Raman-based diagnostic approach to SSC. ${ }^{11}$ The quantification of relative water signals with Raman spectroscopy within and surrounding the tumor in squamous cell carcinoma tissues (54-57\% water in normal tissue margins vs $76 \%$ in the tumor $)^{11}$ can provide clinicians with real-time information on the adequacy of the surgical margin and improve patient outcomes. One of the key advantages of Raman water measurements is the almost complete separation

Received: April 29, 2020

Accepted: June 30, 2020

Published: June 30, 2020 
of the Raman water peak $\left(3390 \mathrm{~cm}^{-1}\right)$ from the other Raman bands of biological constituents, including the $\mathrm{N}-\mathrm{H}$ peak. ${ }^{12}$ The peak is broad and relatively very strong and also contains information on the relative contents of bound and free water. ${ }^{12}$ A recent review outlines other potential aspects of Raman spectroscopy applied to surgical tumor margins. ${ }^{13}$ The approach may be valid for breast malignancies as well. Evidence has emerged based on the other investigative methods, such as magnetic resonance spectroscopy ${ }^{14}$ and imaging, ${ }^{15}$ terahertz imaging, ${ }^{16}$ and diffuse optical spectroscopy. ${ }^{5,17}$ Independent research findings indicate a difference in the water concentration of around $20 \%$ between the tumor and the surrounding breast tissue. ${ }^{18,19}$ Conventional backscattering Raman spectroscopy can only probe very shallow sample layers (typically hundreds of micrometers in biological tissues). In contrast, transmission Raman spectroscopy was shown to be capable of interrogating depths of up to several centimeters in tissue and as such provides a unique capability for probing deep inside tissue noninvasively, including a potential future in in vivo diagnostics. Various studies have been carried out in recent years demonstrating the capability of deep Raman spectroscopy to monitor subsurface components in biological samples several centimeters deep, ${ }^{20,21}$ clearly holding a noninvasive advantage over alternative approaches, such as relying on Raman needle probes developed for subcutaneous use. ${ }^{8}$ Considering these and other previous observations of the contrast in water content and given the unique capability of deep Raman spectroscopy to probe tissue at depth, we have decided to carry out a series of proof-of-principle experiments to assess its potential for the noninvasive detection of waterrich regions and the measurement of the relative water content within tissues at depth. This method has the potential to provide a transcutaneous and noninvasive in vivo cancer diagnosis.

Here we demonstrate for the first time the capability of deep Raman spectroscopy to detect water-rich regions at depth, noninvasively, within bulk tissue with different water concentrations. This shows the potential to rapidly identify suspected zones in a sample for the presence of cancers. These areas could then be further probed in the Raman fingerprint region (with longer acquisition times) to identify chemically specific biomarkers of cancer lesions to confirm their presence and enable their classification.

The set up used in these experiments is a modified transmission Raman spectroscopy (TRS) instrument (Figure 1) built around a $680 \mathrm{~nm}$ wavelength-stabilized laser (IPS). The illumination wavelength was selected to enable the high-

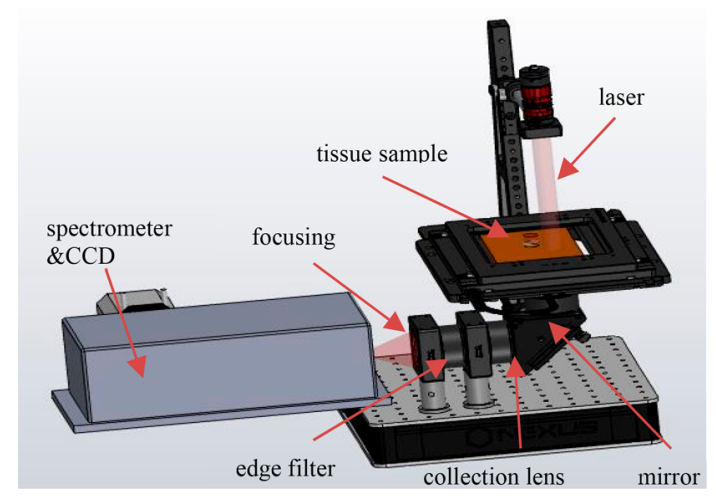

Figure 1. Schematics of the transmission Raman spectroscopy setup. efficiency detection of Raman bands of water spectra found in the high-wavenumber region, when using a silicon-based CCD detector. These generally have a limited sensitivity beyond $1000 \mathrm{~nm}$ and also considering the relatively low absorption of tissue at $680 \mathrm{~nm}$. The laser beam was delivered via a multimode fiber to an FC/PC-terminated zoom fiber collimator to form a beam $5 \mathrm{~mm}$ in diameter, which was spectrally filtered (680 nm laser band-pass filter, Thorlabs) and directed onto the sample $(160 \mathrm{~mW})$. Any subsequent heating by laser irradiation was deemed negligible given the low laser irradiances used $\left(8.1 \mathrm{~mW} / \mathrm{mm}^{2}\right)$ and having previously demonstrated this in-house using IR thermal cameras. As such, we do not envision temperature change to cause any significant spectral changes. Furthermore, all the experiments were performed in temperature and humidity controlled laboratories with tissue samples held at equilibrated room temperature. The temperature variation within lab premises was controlled to $\pm 1{ }^{\circ} \mathrm{C}$.

The Raman signal was collected using $50 \mathrm{~mm}$ diameter optical elements. A $38.8 \mathrm{~mm}$ focal length lens was followed by spectral edge long-pass filters (Semrock Razor Edge, $830 \mathrm{~nm}$ ) eliminating the elastically scattered light and a lens with a 75 $\mathrm{mm}$ focal length imaging the sample collection zone on the spectrograph slit matching its numerical aperture of 0.27 (Holospec 1.8i, Kaiser Optical). For Raman shifted light at $2700 \mathrm{~cm}^{-1}$, the equivalent wavelength is around $832 \mathrm{~nm}$ for a $680 \mathrm{~nm}$ excitation wavelength, which makes the $830 \mathrm{~nm}$ longpass edge filter very convenient for the laser cutoff.

We used fresh pork meat with a high content of muscle tissue from a local butcher as opposed to supermarket samples to avoid any potential content alteration by additional chemical processing, e.g., added water and preservatives. The thickness of the samples was dictated by the cutting process performed by the local butcher.

In the first set of measurements, we monitored the kinetics of a natural drying at room temperature for a tissue block 5 $\mathrm{mm}$ thick (pork muscle) over a time span of $12 \mathrm{~h}$. Tissue weight loss, ascribed to reduction of water content, was monitored by electronic scales with time-stamped data points digitally recorded in parallel with Raman spectra. The aim was to establish that the transmission Raman system is capable of detecting and quantifying tissue water content with adequate sensitivity. In these measurements, the sample was placed on a 3D printed holder frame to ensure the stability of the tissue during the measurement of a time series, with enough clearance on both sides of the tissue sample for the incident laser beam and the collection of Raman photons.

The second part of the study included the measurements of porcine tissue spiked at its center with extra water. To facilitate this, a fresh sample was cut to the approximate dimensions of $51 \times 39 \times 12 \mathrm{~mm}$. A cylinder with a $20 \mathrm{~mm}$ diameter was scooped from the sample and roughly situated in the middle of the scanned region (Figure 2). Once extracted, the cylinder was sliced into 3 smaller cylindrical elements (Figure 2), and the central element (the "core") with a thickness of around 5 $\mathrm{mm}$ was injected with additional water equivalent to an extra $20 \%$ of its weight and wrapped in cling film to prevent water leakage into surrounding zones. The added water increased the water content in the spiked tissue core from approximately 70 to $75 \%$. Note, all calculations in this paper are based around an assumption that the muscle tissue contained around $70 \%$ water before spiking. The cylinder was then reassembled and inserted back in the sample to its original location. Transmission 

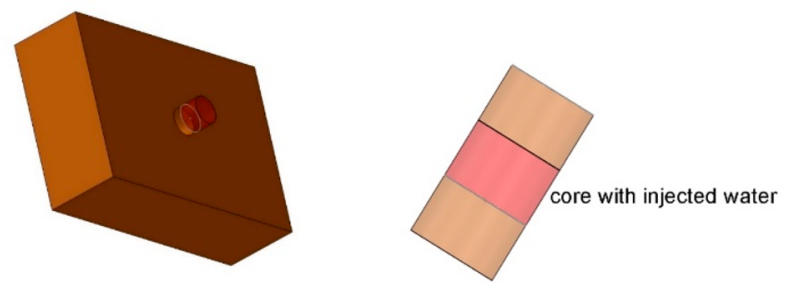

Figure 2. Drawings illustrating the extracted core (magnified, right image) and the location of the water-spiked core within the larger tissue block (left image). The original size of the mapped tissue block was $51 \times 39 \times 12 \mathrm{~mm}$. The cylinder had a $20 \mathrm{~mm}$ diameter and was made up of a spiked core region $5 \mathrm{~mm}$ thick surrounded by unspiked regions $3-3.5 \mathrm{~mm}$ thick.

Raman spectral maps were acquired noninvasively across this assembly before and after the water spiking.

The samples were placed on an automated $x y$ positioning stage (Standa, Vilnius, Lithuania) to permit the spectral mapping. The maps were formed by raster scanning the sample with a $3 \mathrm{~mm}$ step size over an area of $\sim 20 \mathrm{~cm}^{2}$ of the sample and using an acquisition time per map point of $1 \mathrm{~s}$ with 5 accumulations (i.e., $5 \mathrm{~s}$ total per point).

Following acquisition, raw data were imported in Matlab (Matworks) for pre-processing and analysis. The first step in data preprocessing consisted of manual removal of any cosmic ray signals. It was followed by a third-order polynomial baseline subtraction of the entire spectrum and the subsequent normalization to the $\mathrm{CH}$ Raman peak area. Standard Matlab functions were used for the spectral peak analysis, followed by image reconstruction such as computing the selected peak area ${ }^{7}$ of the $\mathrm{OH}$ Raman bands covering spectral region from $3350-3550 \mathrm{~cm}^{-1}$. The main water peak at $3390 \mathrm{~cm}^{-1}$ (which corresponds to unbound water ${ }^{22}$ ) was used to assess the change in the water content because water changes from the tumor tissue are most likely to be in unbound water as they originate from leaky neovasculature. This region was selected to avoid the $3250 \mathrm{~cm}^{-1}$ peak (which corresponds to bound water ${ }^{22}$ ), which overlaps with a peak corresponding to $\mathrm{NH}$ vibrations from proteins and so would give inaccuracies to the change in water content. ${ }^{23}$ By using the peak $\sim 3390 \mathrm{~cm}^{-1}$ we avoided any interaction with these peaks and so achieved a more accurate measurement of the change in water content.

Figure 3 depicts representative snapshots in the evolution of the Raman spectra of the tissue while drying at room temperature. The plotted spectra are normalized to the $\mathrm{CH}$ band. Three spectra are presented, one captured at the initial tissue weight and two at 17.5 and $32 \%$ reductions in tissue sample weight (assumed to be entirely due to water loss). The

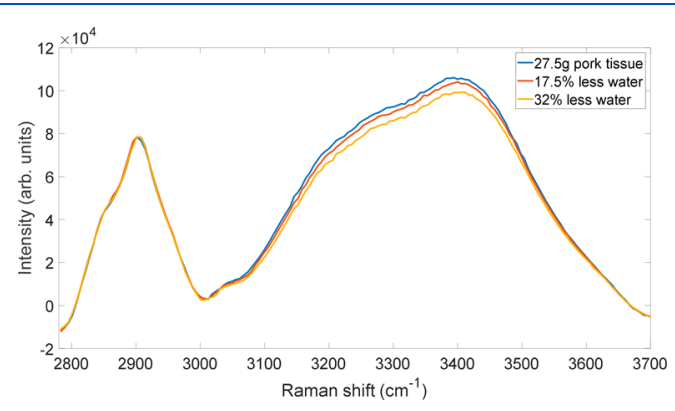

Figure 3. Raman spectra of a drying pork tissue sample with a varying water content over a time span of $12 \mathrm{~h}$. water percentage calculations are based on the net weight loss attributed to water evaporation in the tissue and are correlated with the time stamp of the Raman spectra. The range of water peaks between 3350 and $3550 \mathrm{~cm}^{-1}$ were used to calculate the peak area of the $\mathrm{OH}$ peak. This indicated a change of only $4.5 \%$ associated with $17.5 \%$ real mass loss and of $10 \%$ related to $32 \%$ real mass loss. This discrepancy is attributed to the fact that evaporation occurs predominantly from surface areas of the sample in conjunction with transmission Raman spectroscopy being the least sensitive to surface zones due to its inherent (typically several fold) bias toward central and inner parts of sample as opposed to very near-surface areas in this sampling geometry.

The Raman map of the tissue block containing the cylindrical tissue assembly (Figure 4a), before water spiking

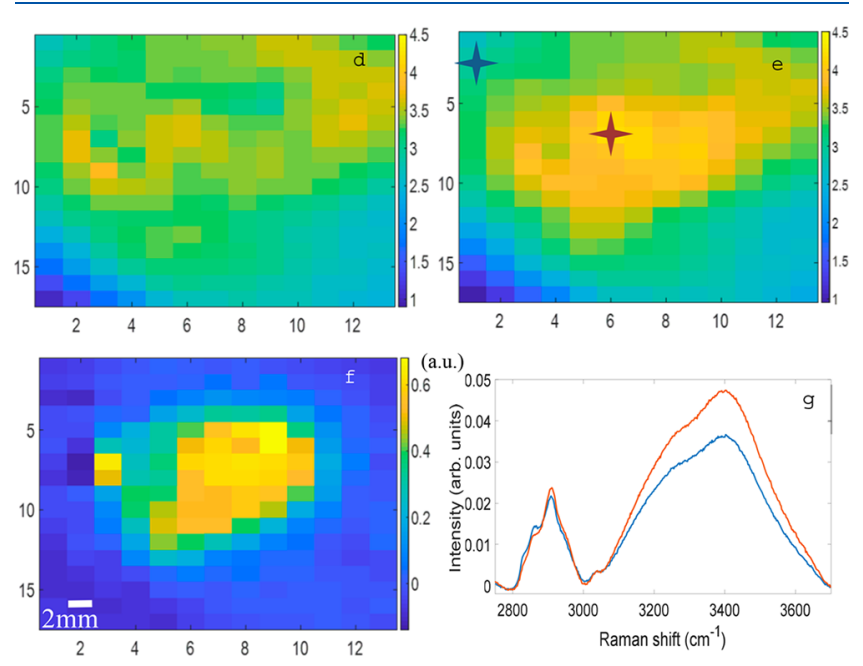

Figure 4. False color maps depicting the Raman water distribution (the area of the selected region of the $\mathrm{OH}$ Raman bands, normalized to the $\mathrm{CH}$ area in the spectrum, in arbitrary units) inside porcine sample with $2 \mathrm{~mm}$ step size in each direction a) prior to injection b) after injection c) difference map (before subtracted from after). Map size is $17 \times 13$ pixels. d) example of Raman spectra from two locations of map in Figure $4 \mathrm{~b}$ (the star color matches the plot color).

the core, illustrates the initial heterogeneity of the tissue's water distribution across the mapped zone. The Raman signals we obtained in this study of the $\mathrm{CH}$ region suggest that the meat measured was predominantly protein-rich tissue. Figure $4 \mathrm{~d}$ does show a small difference in the $2800-2900 \mathrm{~cm}^{-1}$ region, indicating a subtle change in the relative lipid content between the regions indicated by the stars in Figure $4 \mathrm{~b}$. This gives us confidence that the samples were predominantly protein rich meat with negligible amounts of lipids. Even so, by integrating over the whole $\mathrm{CH}$ region between 2800 and 3000 $\mathrm{cm}^{-1}$ we were able to take these contributions into account in our analysis.

After this scan, the middle $5 \mathrm{~mm}$ disk of the removed cylinder was injected with water, equivalent to a $20 \%$ weight increase (effectively raising the water concentration in the tissue from $70^{26}$ to $75 \%$ ) and loosely representing the presence of a cancerous lesion, ${ }^{5}$ which might contain even larger increases in the amount of water (as much as $20 \%$ increase in total water, i.e., from 50 to $70 \%$ water by mass). We assume that after injection the core will contain a higher amount of free water in the sample. A second scan (Figure 4b) was then performed on the tissue block containing the cylindrical tissue 
assembly, now containing the spiked core using the same parameters as used previously. The maps shown in Figure 4 a and $b$ were obtained by computing the selected Raman water band area $(\mathrm{OH})$ after being normalized to the Raman band area of protein and lipids $(\mathrm{CH})$. An example of two Raman spectra (Figure 4d) from different locations of the map in Figure $4 \mathrm{~b}$ is indicative of the range in $\mathrm{OH}$ peak evolution over the entire area of the sample. The signal contrast between Figure $4 \mathrm{a}$ (before) and Figure $4 \mathrm{~b}$ (after spiking) is naturally small because the total amount of water added is relatively small compared to that of the overall water in the bulk sample; however, the presence of the added water is clearly revealed by subtracting the maps from each other (Figure 4c).

The analysis of the average pixel value from the central zone, encompassing the region of maximum signal ( 9 pixels, Figure 5

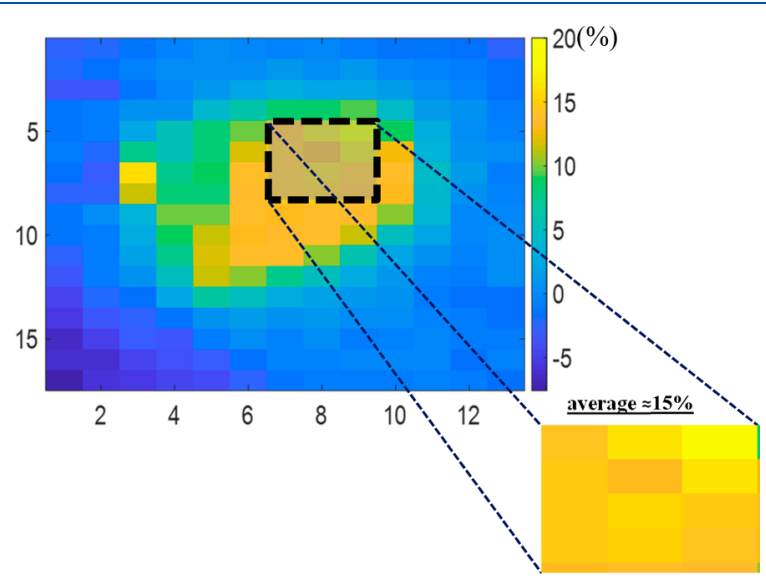

Figure 5. Difference Raman water map from Figure 4 plotted in terms of the water percentage change $\left(17 \times 13\right.$ pixels, pixel size $\left.2 \times 2 \mathrm{~mm}^{2}\right)$ and a zoom into the inner $9 \times 9$ pixel zone (insert).

insert), indicates an average relative water elevation of $15 \%$ in the cylinder assembly as a whole (as TRS probes the full depth of the tissue). This value was obtained as a percentage of the ratio between values in panels a and $c$ of Figure 4. As the spiked middle core represents only $\sim 40 \%$ of the entire cylinder volume, which is measured in its entirety with TRS, this value translates to a $38 \%(0.15 / 0.4=0.38)$ increase in water mass in the spiked core volume (not taking into account any potential TRS signal bias at specific depths). This equates to a calculated water concentration in the spiked core of $76 \%$ when taking into account the increased total mass of the central volume of the spiking water (as well as the original tissue mass); see the Supporting Information for details. This matches remarkably well with the known water-spiked tissue concentration of around $75 \%$ in tissue. This is broadly consistent because the precision of the cylinder slice thicknesses was $\pm 0.5 \mathrm{~mm}$, thus affecting the relative volume of the spiked core to the cylinder assembly as a whole. Furthermore, there is an expected bias of TRS toward the central parts of the sample, ${ }^{24}$ resulting in a higher signal from the central volumes and a slightly lower signal closer to the surfaces. This, if accounted for, would further decrease the predicted value. This proof-of-concept result conceptually demonstrates the basic capability of TRS to detect, by noninvasive means, a relatively large lesion based on the elevated water concentration in this type of sample.

Here, we have conceptually demonstrated the detection of a spiked tissue core with a volume of approximately $1.5 \mathrm{~cm}^{3}$, with the concentration of water in that tissue raised from 70 to
$75 \%$. In cancers, the water concentration increase can be as much as $20 \%$ when transitioning from normal to cancerous tissues. Should the concentration be increased by this much above the background normal tissue water concentration, the spiked core or "tumor" could be reduced in volume by a factor of 4 to yield a similar result. This could equate to a spherical lesion of approximately $9 \mathrm{~mm}$ in diameter buried within the sampling volume. This is the first proof of concept of this approach, and the sensitivity would be expected to improve with appropriate system optimization.

In summary, we have conceptually demonstrated the capability of TRS to noninvasively detect elevated levels of water buried deep inside biological tissue. Our proof-ofconcept setup was able to measure differences in water concentration of $17.5 \%$ when a block of tissue undergoes slow drying at room temperature. When evaluating the ability to detect a "concealed lesion" zone at depth with an artificially elevated water concentration (of 5\% water to tissue concentration in the spiked volume), the technique was able to distinguish, quantify, and clearly image the water-rich region within the sample. The results pave the way to explore the potential for rapid and noninvasive in vivo localization of cancer lesions, which naturally contain a higher water content (mostly driven by leaky neovasculature found in tumors).

\section{ASSOCIATED CONTENT}

\section{SI Supporting Information}

The Supporting Information is available free of charge at https://pubs.acs.org/doi/10.1021/acs.analchem.0c01842.

Calculation of the water percentage in the tissue in the spiked core based on the measured Raman spectra (PDF)

\section{AUTHOR INFORMATION}

\section{Corresponding Authors}

Nicholas Stone - Biomedical Spectroscopy Lab, School of Physics and Astronomy, College of Engineering, Mathematics and Physical Sciences, University of Exeter, Exeter EX4 4QL, U.K.; Institute of Biomedical and Clinical Science, University of Exeter Medical School, Exeter EX1 2LU, U.K.; Royal Devon and Exeter Hospital, Exeter EX2 5DW, U.K.; orcid.org/ 0000-0001-5603-3731; Email: n.stone@exeter.ac.uk

Pavel Matousek - Central Laser Facility, Research Complex at Harwell, STFC Rutherford Appleton Laboratory, Oxfordshire OX110QX, U.K.; Email: pavel.matousek@stfc.ac.uk

\section{Authors}

Adrian Ghita - Biomedical Spectroscopy Lab, School of Physics and Astronomy, College of Engineering, Mathematics and Physical Sciences, University of Exeter, Exeter EX4 4QL, U.K.

Thomas Hubbard - Institute of Biomedical and Clinical Science, University of Exeter Medical School, Exeter EX1 2LU, U.K.; Royal Devon and Exeter Hospital, Exeter EX2 5DW, U.K.

Complete contact information is available at:

https://pubs.acs.org/10.1021/acs.analchem.0c01842

\section{Author Contributions}

A.G. contributed to the instrumentation design and construction; the sample preparation; data measurements, the analysis and interpretation; and paper write up. T.H. contributed to the data interpretation and the paper write 
up. P.M. and N.S. contributed to obtaining the grant funding for the project, the design of the study, the data interpretation, and the paper write up.

\section{Notes}

The authors declare no competing financial interest.

Data from this paper can be obtained by emailing the corresponding authors.

\section{ACKNOWLEDGMENTS}

This work was supported by the Engineering and Physical Sciences Research Council under Grant EP/P012442/1.

\section{REFERENCES}

(1) Quarto, G.; Spinelli, L.; Pifferi, A.; Torricelli, A.; Cubeddu, R.; Abbate, F.; Balestreri, N.; Menna, S.; Cassano, E.; Taroni, P. Biomed. Opt. Express 2014, 5 (10), 3684.

(2) Paran, Y.; Bendel, P.; Margalit, R.; Degani, H. NMR Biomed. 2004, 17 (4), 170-180.

(3) Saadoun, S.; Papadopoulos, M. C.; Davies, D. C.; Krishna, S. Aquaporin-4 Expression Is Increased in Oedematous Human Brain Tumors; Vol. 72; 2002.

(4) Dixon, W. T. Radiology 1984, 153 (1), 189-194.

(5) Chung, S. H.; Yu, H.; Su, M.-Y.; Cerussi, A. E.; Tromberg, B. J. J. Biomed. Opt. 2012, 17 (7), No. 071304.

(6) Boas, D.A.; Brooks, D.H.; Miller, E.L.; DiMarzio, C.A.; Kilmer, M.; Gaudette, R.J.; Zhang, Q. IEEE Signal Process. Mag. 2001, 18 (6), 57-75.

(7) Caspers, P. J.; Bruining, H. A.; Puppels, G. J.; Lucassen, G. W.; Carter, E. A. J. Invest. Dermatol. 2001, 116 (3), 434-442.

(8) Day, J. C. C.; Bennett, R.; Smith, B.; Kendall, C.; Hutchings, J.; Meaden, G. M.; Born, C.; Yu, S.; Stone, N. Phys. Med. Biol. 2009, 54 (23), 7077-7087.

(9) Fisher, S.; Yasui, Y.; Dabbs, K.; Winget, M. BMC Health Serv. Res. 2018, 18 (1), 94.

(10) Dixon, J. M.; Newlands, C.; Dodds, C.; Thomas, J.; Williams, L. J.; Kunkler, I. H.; Bing, A.; Macaskill, E. J. Br. J. Surg. 2016, 103 (7), 830-838.

(11) Barroso, E. M.; Smits, R. W. H.; van Lanschot, C. G. F.; Caspers, P. J.; Ten Hove, I.; Mast, H.; Sewnaik, A.; Hardillo, J. A.; Meeuwis, C. A.; Verdijk, R.; Noordhoek Hegt, V.; Baatenburg de Jong, R. J.; Wolvius, E. B.; Bakker Schut, T. C.; Koljenović, S.; Puppels, G. J. Cancer Res. 2016, 76 (20), 5945-5953.

(12) Leikin, S.; Parsegian, V. A.; Yang, W.-H.; Walrafen, G. E. Proc. Natl. Acad. Sci. U. S. A. 1997, 94, 11312-11317.

(13) Hubbard, T. J. E.; Shore, A.; Stone, N. Analyst 2019, 144 (22), 6479-6496.

(14) Agarwal, K.; Sharma, U.; Mathur, S.; Seenu, V.; Parshad, R.; Jagannathan, N. R. Magn. Reson. Imaging 2018, 49, 116-122.

(15) Thakur, S. B.; Brennan, S. B.; Ishill, N. M.; Morris, E. A.; Liberman, L.; Dershaw, D. D.; Bartella, L.; Koutcher, J. A.; Huang, W. J. Magn. Reson. Imaging 2011, 33 (4), 855-863.

(16) Fitzgerald, A. J.; Wallace, V. P.; Jimenez-Linan, M.; Bobrow, L.; Pye, R. J.; Purushotham, A. D.; Arnone, D. D. Radiology 2006, 239 (2), 533-540.

(17) Chung, S. H.; Cerussi, A. E.; Klifa, C.; Baek, H. M.; Birgul, O.; Gulsen, G.; Merritt, S. I.; Hsiang, D.; Tromberg, B. J. Phys. Med. Biol. 2008, 53 (23), 6713-6727.

(18) Carpenter, C. M.; Pogue, B. W.; Jiang, S.; Dehghani, H.; Wang, X.; Paulsen, K. D.; Wells, W. A.; Forero, J.; Kogel, C.; Weaver, J. B.; Poplack, S. P.; Kaufman, P. A. Opt. Lett. 2007, 32 (8), 933.

(19) Cerussi, A.; Hsiang, D.; Shah, N.; Mehta, R.; Durkin, A.; Butler, J.; Tromberg, B. J. Proc. Natl. Acad. Sci. U. S. A. 2007, 104 (10), 4014-4019.

(20) Matousek, P.; Stone, N. Chem. Soc. Rev. 2016, 45 (7), 17941802.

(21) Ghita, A.; Matousek, P.; Stone, N. J. Biophotonics 2018, 11 (1), No. e201600260.
(22) Unal, M.; Akkus, O. Bone 2015, 81, 315-326.

(23) Yang, S.; Sen, C.; Zhou, J. InGaAs Detector Based Raman Spectroscopy for Water Probing in Biological Tissue. In Biophysics, Biology and Biophotonics IV: the Crossroads; Wax, A., Backman, V., Eds.; Vol. 10888; SPIE: Bellingham, WA, 2019; p 19.

(24) Matousek, P.; Everall, N.; Littlejohn, D.; Nordon, A.; Bloomfield, M. Appl. Spectrosc. 2011, 65 (7), 724-733.

(25) Owen, H.; Lewis, I.; Priestnall, I.; Andrews, J.; Davis, K.; George, M. W.; Everall, N.; Dallin, P. Appl. Spectrosc. 2010, 64 (5), 476-484.

(26) U.S. Department of Agriculture, Food Safety and Inspection Service. Water in Meat and Poultry. https://www.fsis.usda.gov/wps/ portal/fsis/topics/food-safety-education/get-answers/food-safetyfact-sheets/meat-preparation/water-in-meat-and-poultry/ct_index. 